\title{
MATRIX METHODS OF SUMMATION, REGULAR FOR p-ADIC VALUATIONS
}

\author{
RICHARD V. ANDREE AND GORDON M. PETERSEN
}

A method of summation for sequences is defined to be regular if it "sums" every convergent sequence (with respect to the absolute value) to its sum. In 1910-1913 Silverman and Töplitz discovered necessary and sufficient conditions for matrix methods of summation to be regular with respect to absolute value. The theory of divergent series has expanded and many useful methods have been investigated, Hardy [1]. Matrices with similar properties for sequences which converge with respect to the $p$-adic valuations are investigated in this paper. The principal result is a set of necessary and sufficient conditions such that the matrix $M_{p k}$ defines a summation method which is regular with respect to the $p$-adic valuation $\phi_{p}$.

Whenever it is unnecessary to distinguish between $\phi_{p}$ and $\phi_{q}$, the symbol $\phi$ will be used to denote a $p$-adic valuation, $\phi_{p}$, of the rational field with respect to a prime $p$.

Definition 1. A sequence $\left\{t_{i}\right\}$ obtained from the matrix $\left[a_{m n}\right]$ and the sequence $\left\{S_{i}\right\}$ using the relationship $t_{m}=\sum_{i=1}^{m} a_{m i} S_{i}$ is said to be the $\left[a_{m n}\right]$ transform of $\left\{S_{i}\right\}$. If the sequence $\left\{t_{i}\right\}$ converges to $T$, the matrix $\left[a_{m n}\right]$ is said to sum the sequence $\left\{S_{i}\right\}$ to the sum $T$.

Definition 2. The method of summation defined by the matrix $\left[a_{m n}\right]$ is called regular in the $p$-adic field $\Omega_{p}$ if every convergent sequence $\left\{S_{i}\right\}$ is equal to its transform $\left\{t_{i}\right\}$ in $\Omega_{p}$. Sequences $\left\{t_{i}\right\}$ and $\left\{S_{i}\right\}$ are, of course, equal if $\lim _{m \rightarrow \infty} \phi\left(t_{m}-S_{m}\right)=0$.

Clearly, if $\left\{S_{i}\right\}$ is $p$-convergent, then $\left\{t_{i}\right\}$ is $p$-convergent. The converse need not hold.

Lemma. If $\phi_{p}(a)=p^{-n}, n \geqq 1$, and $\phi(b)=p^{+m}, m \geqq 1$, then $\phi(a+b)$ $=p^{m}$.

Proof. $a=\left(r_{1} / s_{1}\right) p^{n}, b=\left(r_{2} / s_{2}\right) p^{-m}$, with $r_{2}, s_{1}$ prime to $p$ and $(m+n) \geqq 2$. Since $p \nmid r_{2} s_{1}$ and $(m+n) \geqq 2, \phi(a+b)=p^{m}$.

ThEOREM. The three conditions

$$
\lim _{m \rightarrow \infty} \phi\left(a_{m k}\right)=0,
$$

Received by the editors June 14, 1954 and, in revised form, March 28, 1955 and May 6, 1955. 


$$
\begin{aligned}
\lim _{m \rightarrow \infty} \phi\left(\sum_{i=0}^{m} a_{m i}-1\right) & =0, \\
\phi\left(a_{m n}\right) & \leqq M,
\end{aligned}
$$

are necessary and sufficient conditions that the method defined by $t_{m}=\sum_{i=0}^{m} a_{m i} S_{i}$ be regular- $p$.

Proof. The proofs of the necessity of conditions (1) and (2) are similar to the classic case, mutatis mutandis. They will not be reproduced here. The proofs of the necessity of condition (3) and of the sufficiency follow.

Suppose that condition (3) is not satisfied; then for $m=m_{1}, m_{2}, \ldots$

$$
\phi_{p}\left(a_{m n_{m}}\right)=p^{\alpha_{m}}
$$

where $\alpha_{m_{\nu}}>N, m_{\nu}>M$. We can assume without prejudice to our construction in the sequel that $m_{1}=1, m_{2}=2, \cdots$, etc., and so simplify our notation. By $a_{m n_{m}}$ we shall denote the first $a_{m n}$ in a row $m$ such that $\phi_{p}\left(a_{m n}\right)=p^{\alpha_{m}}$. Since condition (1) is necessary, it is evident that $n_{m}>\nu$ whenever $m>M$. Now choose a sequence $\left\{\mu_{i}\right\}$ such that $n_{\mu_{i}}>\mu_{i-1}$ and $\phi\left(a_{\mu_{i} n_{\mu_{i}-1}} p^{\alpha_{\mu_{i-1}}-2}\right)<1 / p^{2}$. Let $S_{n}=0, n \neq \mu_{i}$ and $S_{n_{\mu_{i}}}$ $=p^{\alpha \mu_{i}-2}$.

It is clear that

$$
\phi\left(\sum_{n=0}^{n_{\mu_{i}}-1} a_{\mu_{i} n} S_{n}\right)<\frac{1}{p^{2}}, \quad \phi\left(\sum_{n=n_{i}}^{\mu_{i}} a_{\mu_{i} n} S_{n}\right)=p^{2},
$$

and so by our lemma

$$
\phi\left(t_{\mu_{i}}-S_{\mu_{i}}\right)=p^{2} .
$$

However, $\lim _{n \rightarrow \infty} \phi\left(S_{n}-S_{m}\right)=0$. Hence, condition (3) is necessary.

To prove the conditions sufficient, it will be shown that if the sequence $\left\{S_{m}\right\}$ converges in the $p$-adic valuation, then $\left\{t_{m}\right\}$ and $\left\{S_{m}\right\}$ belong to the same residue class, i.e. $\lim _{m \rightarrow \infty} \phi\left(t_{m}-S_{m}\right)=0$.

$$
\begin{aligned}
& \phi\left(t_{m}-S_{m}\right)=\phi\left(\sum_{n=0}^{m} a_{m n} S_{n}-S_{m}\right) \\
&=\phi\left(\sum_{n=0}^{m} a_{m n}\left[S_{n}-S_{m}\right]+\sum_{n=0}^{m} a_{m n} S_{m}-S_{m}\right) \\
& \leqq \phi\left(\sum_{n=0}^{m} a_{m n}\left[S_{n}-S_{m}\right]\right)+\phi\left(\left[\sum_{n=0}^{m} a_{n m}-1\right] S_{m}\right) . \\
& \phi\left(\left[\sum_{n=0}^{m} a_{n m}-1\right] S_{m}=\phi\left(S_{m}\right) \phi\left(\sum_{n=0}^{m} a_{n m}-1\right) .\right.
\end{aligned}
$$


Since $\phi\left(S_{n}\right)$ is bounded, by condition (2)

$$
\phi\left(\left[\sum_{n=0}^{m} a_{m n}-1\right] S_{m}\right) \leqq C_{1}, \quad m>M_{1} \text {. }
$$

Also if $m, n>\nu, \phi\left(S_{m}-S_{n}\right)<\epsilon$. Hence,

$$
\begin{aligned}
\phi\left(\sum_{n=\theta}^{m} a_{m n}\left[S_{n}-S_{m}\right]\right) \leqq \phi & \left(\sum_{n=0}^{\infty} a_{m n}\left[S_{n}-S_{m}\right]\right) \\
& +\phi\left(\sum_{\nu+1}^{m} a_{m n}\left[S_{n}-S_{m}\right]\right)
\end{aligned}
$$

and

$$
\begin{aligned}
\phi\left(\sum_{\nu+1}^{m} a_{m n}\left[S_{n}-S_{m}\right]\right) & \leqq \operatorname{Max}_{i \geq \nu+1} \phi\left(a_{m i}\left[S_{i}-S_{m}\right]\right) \\
& \leqq \operatorname{Max}_{i \geqq v+1} \phi\left(a_{m i}\right) \underset{i \geq \nu+1}{\operatorname{Max}} \phi\left(S_{i}-S_{m}\right) \\
& \leqq C_{2} \epsilon .
\end{aligned}
$$

Now we shall keep $\nu$ fixed but shall choose $m=m_{1}>M_{3} \geqq \nu$ so that $\operatorname{Max}_{i \leqq \nu} \phi\left(a_{m_{i} n}\right)<\epsilon$. Of course, it will still be true that $m_{1}>\nu$ and $\operatorname{Max}_{i \geqq v+1}\left(S_{i}-S_{m_{1}}\right)<\epsilon$.

Then

$$
\begin{aligned}
\phi\left(\sum_{n=0}^{\nu} a_{m_{1} n}\left[S_{n}-S_{m_{1}}\right]\right) & \leqq \operatorname{Max}_{t \leqq \nu} \phi\left(a_{m_{1} n}\left[S_{n}-S_{m_{1}}\right]\right) \\
& \leqq \operatorname{Max}_{t \leqq \nu} \phi\left(a_{m_{1} n}\right) \operatorname{Max} \phi\left(S_{n}-S_{m_{1}}\right) \leqq C_{3} \epsilon .
\end{aligned}
$$

Hence

$$
\phi\left(t_{m_{1}}-S_{m_{1}}\right) \leqq C_{1} \epsilon+C_{2} \epsilon+C_{3} \epsilon
$$

or

$$
\lim _{m \rightarrow \infty} \phi\left(t_{m}-S_{m}\right)=0 .
$$

This theorem is similar to the theorem of Silverman and Töplitz, Hardy [1]. The parallel would be exact if condition (3) were replaced by the stronger condition

$$
\sum_{n=0}^{m} \phi\left(a_{m n}\right) \leqq M
$$

Condition ( $\left.3^{\prime}\right)$ implies (3) and so is sufficient; however, it is not nec- 
essary. This can be seen from the regular- $p$ method of summation described below.

If $p^{\nu} \leqq m<p^{\nu+1}$, let $a_{m n}=0, n<m-p^{\nu-1}-2, a_{m n}=1 / p, m-p^{\nu-1}-2$ $\leqq n<m-2, a_{m m}-1=-p^{\nu-2}, a_{m m}=1$. Since

$$
\sum_{n=0}^{m} \phi\left(a_{m n}\right)=p^{\nu}+\frac{1}{p^{n-2}}+1, \quad p^{\nu} \leqq m<p^{\nu+1},
$$

condition $\left(3^{\prime}\right)$ is not satisfied; on the other hand this method satisfies the conditions of the theorem and so is regular- $p$, a fact which easily can be verified directly.

The sufficiency proof holds for non-Archimedian valuations in general. The particular properties of the $p$-adic valuation are used only in the proof of the necessity of (3).

The theorem involves triangular matrices, although formally the proof can be extended to infinite-row matrices. This extension has not been made since the individual $t$ 's of the transform may then become $p$-adic numbers.

There exist $p$-regular matrices $\left[a_{m n}\right]$ which produce $p$-adic convergent sequences $\left\{t_{n}\right\}$ as transforms of $p$-adic divergent sequences $\left\{S_{n}\right\}$. Consider the method $t_{m}=S_{m-1} / 2+S_{m} / 2$. It is regular- $p$ for every $p$ and sums the sequence $1,0,1,0,1,0, \cdots$ to the sequence $1 / 2,1 / 2,1 / 2, \cdots$.

Another regular- $p$ method is defined by $t_{m}=\sum_{n=0}^{m-1}\left(a p^{m-n-1} S_{n}\right)$ $+b S_{m}$ where $\phi\left(\sum_{n=0}^{m-1} a p^{m-n-1}+b-1\right)<\epsilon, m>\nu$.

\section{REFERENCE}

1. G. H. Hardy, Divergent series, Oxford, 1949.

The University OF OrIahoma 